\title{
Elevated serum ferritin level effectively discriminates severity illness and predicts prognosis of COVID-19 patients
}

\section{Peng Cao}

Huazhong University of Science and Technology https://orcid.org/0000-0001-7228-3511

Yuanjue Wu

Huazhong University of Science and Technology

\section{Sanlan Wu}

Huazhong University of Science and Technology

Tingting Wu

Huazhong University of Science and Technology

\section{Qilin Zhang}

Huazhong University of Science and Technology

\section{Rui Zhang}

Huazhong University of Science and Technology

Yongning Lu

Huazhong University of Science and Technology

Yu Zhang ( $\square$ zhangwkp@163.com )

Huazhong University of Science and Technology

\section{Research}

Keywords: Ferritin, coronavirus, pneumonia, COVID-19, prognosis, risk factor

Posted Date: June 2nd, 2020

DOI: https://doi.org/10.21203/rs.3.rs-31914/v1

License: (a) This work is licensed under a Creative Commons Attribution 4.0 International License. Read Full License 


\section{Abstract}

During coronavirus disease 2019 (COVID-19) pandemic, medical resources in every country is in shortage. Efficacious indicators of discriminating severe illness and predicting outcome is in urgent need.

We collected data and clinical records from 79 COVID-19 patients admitted between January 12, 2020 and February 21, 2020 at Wuhan Union hospital, China. Spearman's correlation analysis, receiver operating characteristic (ROC) curve, logistic regression model, and KaplanMeier survival curves were employed in the analysis.

Of 79 patients enrolled, 2 died in hospital, 8 were transferred to other hospitals, and 69 were discharged. Patients with elevated ferritin levels $(>200 \mathrm{ng} / \mathrm{mL}$ ) had a higher incidence of severity illness when compared with those with normal ferritin levels $(\leq 200 \mathrm{ng} / \mathrm{mL})(50.0 \% \mathrm{vs}$ 2.9\%). In addition, severity illness manifested significantly higher level of ferritin as compared with non-severe ones (median 921.3 vs $130.7 \mathrm{ng} / \mathrm{mL}, \mathrm{p}<0.001$ ). Furthermore, ferritin could effectively discriminate severity and non-severity, with an area under the ROC curve (AUC) reaching 0.873 (sensitivity $96 \%$, specificity $70 \%$ ), larger than that of age (0.697), C-reactive protein (0.730) and lymphocytes\% (0.717). Combined model incorporating multivariate revealed a similar manner with ferritin alone $(p=0.981)$. Furthermore, elevated ferritin group showed longer viral clearance time (median 16 vs 6 days, $p<0.001$ ) and in-hospital length (median 18 vs 10 days, $p<0.001$ ).

Our results suggest that ferritin could act as a simple and efficacious complementary tool to identify severe COVID-19 patients at early stage and predict their outcome. This indicator would provide guidance for subsequent clinical practice, alleviate the medical stress and reduce the mortality.

\section{Introduction}

The coronavirus disease 2019 (COVID-19) pandemic has spread to 44 countries on all continents except Antarctica and the number of diagnosed cases worldwide is accelerating every day [1]. Medical resources in every country are in shortage or even overdrawn, so there is an urgent need of seeking indicators for disease severity and prognosis at early stage, which is believed to significantly reduce the medical stress and mortality rate. However, such efficient indicators have not been fully investigated. Currently, the definition of severe cases mainly relies on the observation of clinical manifestation [2, 3], and the prognosis of COVID-19 remains inconclusive.

Ferritin is the primary tissue iron-storage protein in the liver, it is also an acute-phase protein that can be induced in the setting of systemic inflammation [4]. Ferritin is able to act as an independent predictor of discriminating the severity of nonalcoholic fatty liver disease [5]. Recent studies related to COVID-19 have consistently discovered the abnormal level of ferritin in severe COVID-19 patients, but further exploration of its value is halted [6,7]. Herein, we aimed to assess the ability of ferritin in discriminating severe patients from non-severe ones on admission, and the potential of predicting viral clearance rate and hospitalization duration, to provide guidance for subsequent clinical practice.

\section{Methods}

\subsection{Participants}

From January 12, 2020 to February 21, 2020, a total of 147 consecutive patients were initially enrolled from the department of infectious diseases of Wuhan Union Hospital, all of which were confirmed cases of COVID-19 after examination of COVID-19 RNA by RT-PCR (upper respiratory throat swab samples) and chest computerized tomography (CT) scanning. One patient who died of traumatic brain injury with viral pneumonia was excluded. Patients without ferritin detection on admission were excluded. Therefore, 79 inpatients were enrolled in this study. By March 14, 2 patients died, 8 patients were transferred to other hospitals, and the remaining patients were discharged.

According to "diagnosis and treatment of novel coronavirus pneumonia", severe illness on admission was defined when any of the following criteria is met [8]: 1 . shortness of breath (respire rate $\geq 30$ times $/ \mathrm{min}$ ); 2 . the oxygen saturation is less than $93 \%$ in resting state; 3 . arterial partial pressure of oxygen $\left(\mathrm{PaO}_{2}\right)$ /oxygen concentration $\left(\mathrm{FiO}_{2}\right) \leq 300 \mathrm{mmHg}(1 \mathrm{mmHg}=0.133 \mathrm{kPa})$.

\subsection{Data collection}

The data of demographics, laboratory examinations, CT scan, clinical characteristics, treatments and outcomes were acquired by the hospitalization management system. All the data were collected by well-trained researchers with a double-blind method. Data collection of laboratory results were defined using the first-time examination at admission. The date of symptom onset, admission, negative detection of COVID-19 RNA, discharge and death were recorded accurately. The date of admission was used as the starting point of the virus clearance process, and the date of the negative detection of COVID-19 RNA was calculated as the ending point of viral clearance.

Page $2 / 12$ 


\subsection{Statistical Analysis}

The data were presented as mean \pm standard deviation (SD) or median (Inter Quartile Range, IQR). Intergroup comparisons between groups were made by the Student's $t$ test (normally distributed continuous variables), Mann-Whitney $U$ test (nonnormally distributed continuous variables). The categorical variables (shown by percentage) were compared by using Chi-square analysis. Spearman's correlation analysis was used to explore the coefficients of ferritin with other covariates. The performance of the discriminant model was characterized by estimating the area under the receiver operating characteristic (ROC) curve (AUC). The cutoff value of ROC was calculated based on the maximum Youden index, which was used to assess the global diagnostic effectiveness. Cumulative survival curves of hospitalization span and viral clearance time were estimated using the Kaplan-Meier estimation method for two groups with normal and elevated ferritin levels (ferritin $\leq 200 \mathrm{ng} / \mathrm{mL}$ and ferritin $>200 \mathrm{ng} / \mathrm{mL}$ ) by log-rank test. All of the analyses were performed with the R software version 3.4 .3 (http: //www.R-project.org, The R Foundation) and EmpowerStats version 2.20 (http://www.empowerstats.com, X\&Y Solutions, Inc., Boston, MA). A two-sided $P$ value $<0.05$ was determined as with statistically significant differences.

\section{Results}

\subsection{Baseline data}

All participants were divided into two groups by normal and elevation ferritin levels (ferritin $\leq 200 \mathrm{ng} / \mathrm{mL}$ and ferritin $>200 \mathrm{ng} / \mathrm{mL}$ ) according to clinical reference range. As shown in Table 1, severe cases were more frequently found in patients with higher ferritin levels, compared to those who with normal ferritin levels $(50.0 \%$ vs $2.9 \%, p<0.001)$. There were significant differences of sex between the two groups, with male patients accounting for the $68.2 \%$ in elevation ferritin group but only $17.1 \%$ in normal ferritin group $(p<0.001)$. The average age of elevation ferritin group was also significantly older than the normal ferritin group (49.9 vs 36.8 years old). The other characteristics including systolic blood pressure (SBP), diastolic blood pressure (DBP), respire, pulse, smoking, comorbidities and symptoms of both groups were recorded but no statistical difference was found.

\subsection{Laboratory examination}

As displayed in Supplementary Table 1, the levels of triglyceride (TG), serum amyloid A protein (SAA), alanine aminotransferase (ALT), aspartate aminotransferase (AST), y-glutamyl transferase ( $\mathrm{Y}-\mathrm{GT})$, interleukin-6 (IL-6), creatinine (Cre), lactate dehydrogenase (LDH), fibrinogen (FIB), fibrinogen degradation product (FDP), C-reactive protein (CRP) and erythrocyte sedimentation rate (ESR) in elevation ferritin group were extremely higher than those of normal ferritin group $(p<0.001)$. The levels of fasting blood glucose (FBG), alkaline phosphatase (ALP), natural kill-cell (NK-cell), creatine kinase (CK), troponin (TNI), thrombin time (TT), D-dimer, haemoglobin, leucocytes, neutrophils and neutrophil-to-lymphocyte ratio (NLR) in elevation ferritin group were also higher than those of normal group ( $p<0.05)$. However, the levels of high-density lipoprotein cholesterol (HDL-c), albumin, CD3 + and estimated glomerular filtration rate (eGFR) in elevation ferritin group were significantly lower than those of normal ferritin group $(p<0.05)$. The other indicators showed no insignificance between two groups.

Furthermore, a spearman's correlation analysis was employed to demonstrate the relationship between ferritin and other covariates (Fig. 1). Ferritin was highly positively correlated to FIB, LDH, SAA, Y-GT, NK-cell, FDP, ESR, AST, ALT, CRP, CK, IL-6, Cre and TT ( $r>0.4$ and $p<0.001$ ), and negatively associated with eGFR and CD3 + with a statistical significance $(r<-0.45$ and $p<0.0005)$.

\subsection{Determination of ferritin and corresponding CT manifestation}

As mentioned above, there existed significantly more severe cases in elevation ferritin group than the normal group. In addition, we compared the ferritin levels among severe and non-severe patients, finding that the ferritin level of severe group is significantly higher than that of nonsevere group (median 921.3 (IQR 440.0-1609.8) vs median 130.7 (IQR 58.8-320.4), p < 0.001) (Fig. 2A). Furthermore, we recorded the ferritin levels which were calculated from the onset of symptom of each patient. Likewise, at every stage the ferritin level of sever group was markedly higher than their counterparts (Fig. 2B). On the other side, we observed the CT images that were corresponding to the ferritin values in three representative patients (Fig. 2C). The results demonstrated that the severity of pulmonary imaging was consistent with the ferritin level. Taken together, the ferritin level is able to reveal the severity of COVID-19.

\subsection{Diagnostic ability of ferritin}

The AUC of ROC plot for ferritin was 0.873 (95\% Cl: 0.798-0.954), larger than that of age (0.697, 95\% Cl: 0.567-0.828), CRP (0.730, 95\% Cl: 0.602-0.859) and lymphocytes\% (LYM\%, 0.717, 95\% Cl: 0.586-0.847), indicating ferritin possesses a considerable diagnostic value for severity of patients on admission. The best cutoff value of ferritin was $272.5 \mathrm{ng} / \mathrm{mL}$, with a sensitivity of $96 \%$ and specificity of $70 \%$ (Fig. 3A). Furthermore, we established a multivariable diagnostic model incorporating sex, age, ferritin, LYM\% and CRP ( $\mathrm{n}=69,0.862$ (95\% Cl: $0.761-0.962$ ), sensitivity $77 \%$, specificity $87 \%$ ) (Fig. 3B). It is interesting to find that the diagnostic ability of integrated model showed the 
similar value as ferritin alone with the AUC showing $(n=69,0.861(95 \%$ Cl: $0.773-0.949)$, sensitivity $95 \%$, specificity $68 \%)(p=0.981)$. These results suggest that ferritin may act as an independent risk factor on COVID-19 patients.

In addition, we employed logistic regression analysis to assess the risk factors related with severity illness (Fig. 3C). The results showed that a $100 \mathrm{ng} / \mathrm{mL}$ increase in serum ferritin, the odds ratio (OR) of severity illness was 1.17 (95\% Cl: 1.03-1.33, $\mathrm{p}=0.014$ ). After adjusted for sex, age, CRP or LYM\%, the OR of severity illness was $1.20(95 \% \mathrm{Cl}$. 1.02-1.41, $\mathrm{p}=0.014)$. Moreover, Fig. 3D showed that the OR of severity illness for serum ferritin was $10.78(95 \% \mathrm{Cl}: 1.13-102.73, \mathrm{p}=0.039)$ when serum ferritin was calculated as categorical variable $(>200 \mathrm{ng} / \mathrm{mL} \mathrm{vs} \leq$ $200 \mathrm{ng} / \mathrm{mL}$ ) in crude model. After adjusted for age, sex, CRP and LYM\%, the OR of severity illness for patients with elevated ferritin level (> $200 \mathrm{ng} / \mathrm{mL}$ ) was $18.75(95 \% \mathrm{Cl}: 1.28,275.41, \mathrm{p}=0.032)$, as compared with patients with normal ferritin level $(\leq 200 \mathrm{ng} / \mathrm{mL})$. Therefore, we established a nomogram based on the above risk factors to improve the availability in clinical practice (Fig. 4).

\subsection{Prognostic ability of ferritin}

Median duration of viral clearance was 6 days (IQR 2-25) in normal ferritin group ( $\leq 200 \mathrm{ng} / \mathrm{mL}$, but elevation ferritin group (>200 ng/mL) was 16 days (IQR 2-47). The viral clearance events in in normal group and elevation ferritin group by using Kaplan-Meier survival analysis were displayed in Fig. 5A $(p<0.001)$.

Besides, we recorded the length of hospitalization of all patients except 2 deceased ones and 8 patients that transferred to other hospitals. Our result showed that patients with higher ferritin levels on admission stayed in hospital for a longer time (18 days, IQR 3-47), when compared with the ones with normal ferritin levels (10 days, IQR 5-29). The discharge events in in normal group and elevation ferritin group by using Kaplan-Meier survival analysis are displayed in Fig. 5B $(p<0.001)$.

\section{Discussion}

The number of diagnosed COVID-19 patients worldwide is increasing rapidly every day. Until now, there is no effective medicine available to treat COVID-19. Medical resources in every country are in shortage or even overdrawn, so there is an urgent need of identifying severe cases and poor prognosis cases at early stage of disease. Only in this way, can medical staffs make greater use of limited medical resources and reduce mortality. Recent clinical data have indicated that lymphocyte [9, 10], CRP [11] and other factors may be related to the severity of COVID-19 [6]. However, the role of ferritin seems to be ignored even though clinical observation has discovered the abnormal ferritin levels in severe COVID-19 patients [6]. For the first time, the present study shows the relationships between ferritin and severity and viral clearance in COVID-19 patients.

Herein, the data of 79 patients with COVID-19 pneumonia were analyzed, the baseline characteristics of patients in the severe and non-severe groups were described and compared, and the dynamic changes of ferritin and imaging were demonstrated. Our results showed that ferritin levels differed significantly between severity and non-severity illness. Ferritin variations were also consistent with CT manifestation, presenting the ability to act as a sensitive and accurate indicator. Then, the diagnostic value of ferritin for the incidence of severe illness was compared to that of age, sex, CRP and LYM\%, which had been recognized as the risk factors of COVID-19 [9, 12-14]. Our results showed that the AUC of ferritin ROC were significantly larger than other risk factors, and the multivariable combined model manifested similar ability compared with ferritin alone, demonstrating that ferritin was an independent easy-to-use predictor. In addition, we compared the viral clearance rate and in-hospital length between elevation ferritin group and normal group, finding that ferritin was also a discriminated indictor for prognosis of COVID-19 patients. Viral clearance is the golden standard for defining the recovery of COVID-19 infections and predicting inhospital length is extremely vital in COVID-19 pandemic due to the lack of medical resource [15]. Thus, we suppose ferritin could act as both an effective discriminator for severity illness and a predictor for prognosis.

Ferritin is an acute phase protein which can be released from damaged hepatocytes [16]. Hyperferritinemia has been previously recognized in abnormal liver function conditions or metabolic syndrome $[5,17,18]$. In our study, the severe patients on admission manifested slight risk of liver injury and dyslipidemia. Other observations also find that liver injury is prevalent in severe cases of COVID-19 [19]. Therefore, we recommend that patients should pay close attention to the risk of developing secondary liver diseases or metabolic syndrome after they are discharged. Intensive surveillance and regular comprehensive medical examination are necessary, especially for the severe patients.

However, there were some limitations in the study. First, this was a single-center retrospective study with limited size. Second, some cases had incomplete biochemistry determinations. Third, the present study failed to predict the mortality of COVID-19 patients due to the insufficient number of deaths.

In summary, the findings indicate that early examination of ferritin in COVID-19 patients could effectively discriminate severity illness and predict the prognosis. Therefore, ferritin could act as a simple complementary tool to help guiding clinical decision and facilitating appropriate treatment. Patients with elevated admission ferritin level should be provided with strengthened attention and treatment. More

Page $4 / 12$ 
studies are needed to confirm these findings and to explore exact pathological mechanisms. Further understanding of pathological significance of serum ferritin elevation in severe COVID-19 patients is warranted to help clinicians make reasonable decisions to decrease the risks of adverse outcome.

\section{Conclusion}

In conclusion, serum ferritin might be an independent risk factor for severity illness and predictor for prognosis of COVID-19 patients.

\section{Abbreviations}

ALP: alkaline phosphatase; ALT: alanine aminotransferase; AST: aspartate aminotransferase; AUC: area under the curve; CK: creatine kinase; COVID-19: coronavirus disease 2019; Cre: creatinine; CRP: C-reactive protein; CT: computerized tomography; DBP: diastolic blood pressure; eGFR: estimated glomerular filtration rate; ESR: erythrocyte sedimentation rate; FBG: fasting blood glucose; FDP: fibrinogen degradation product; FIB: fibrinogen; HDL-c: high-density lipoprotein cholesterol; IL-6: interleukin-6; IQR: Inter Quartile Range; LDH: lactate dehydrogenase; NK-cell: natural kill-cell; NLR: neutrophils and neutrophil-to-lymphocyte ratio; OR: odds ratio; ROC: receiver operating curve; SAA: amyloid A protein; SBP: systolic blood pressure; SD: standard deviation; TG: triglyceride; TNI: troponin; TT: thrombin time; $ү$-GT: $y$-glutamyl transferase

\section{Declarations}

\section{Conflicts of interest}

The authors declare no conflicts of interest.

\section{Consent for publication}

Not applicable.

Availability of data and materials

The raw data required to reproduce these findings cannot be shared at this time as the data also forms part of an ongoing study.

\section{Author contributions}

Peng Cao: Data collection, Writing-Original draft preparation, Conceptualization; Yuanjue Wu: Data analysis, manuscript revision; Sanlan Wu: Data collection; Tingting Wu: Data collection; Rui Zhang: Data collection; Qilin Zhang: Data collection; Yu Zhang: Supervision.

\section{Acknowledgments}

We acknowledge all the front-line medical staffs fighting against COVID-19. We show our respect to those who have sacrificed in this war.

\section{Funding}

This study was supported by the National Key R\&D Program of China (2017YFC0909900) and National Natural Science Foundation of China (Grant No. 81903901).

\section{Ethics approval and consent to participate}

This study had been approved by the Ethics Committee of the Union Hospital, Tongji Medical College, Huazhong University of Science and Technology (Review date: March 24, 2020) and was exempted from the need for informed consent from patients because it was a retrospective assessment. All procedures followed the instructions of local ethic committee (approval 2020 NO. 0134).

\section{References}

1. Wu F, Zhao S, Yu B, Chen YM, Wang W, Song ZG, Hu Y, Tao ZW, Tian JH, Pei YY, Yuan ML, Zhang YL, Dai FH, Liu Y, Wang QM, Zheng JJ, Xu L, Holmes EC, Zhang YZ. A new coronavirus associated with human respiratory disease in China. Nature. 2020;579(7798):265-9.

2. Wang D, Hu B, Hu C, Zhu F, Liu X, Zhang J, Wang B, Xiang H, Cheng Z, Xiong Y, Zhao Y, Li Y, Wang X, Peng Z. Clinical Characteristics of 138 Hospitalized Patients With 2019 Novel Coronavirus-Infected Pneumonia in Wuhan, China. JAMA 2020: [Epub ahead of print].

3. Yang X, Yu Y, Xu J, Shu H, Xia J, Liu H, Wu Y, Zhang L, Yu Z, Fang M, Yu T, Wang Y, Pan S, Zou X, Yuan S, Shang Y. Clinical course and outcomes of critically ill patients with SARS-CoV-2 pneumonia in Wuhan, China: a single-centered, retrospective, observational study. 
Lancet Respir Med 2020: [Epub ahead of print].

4. Kalantar-Zadeh K, Rodriguez RA, Humphreys $\mathrm{MH}$. Association between serum ferritin and measures of inflammation, nutrition and iron in haemodialysis patients. Nephrol Dial Transpl. 2004;19(1):141-9.

5. Kowdley KV, Belt P, Wilson LA, Yeh MM, Neuschwander-Tetri BA, Chalasani N, Sanyal AJ, Nelson JE, Network NCR. Serum ferritin is an independent predictor of histologic severity and advanced fibrosis in patients with nonalcoholic fatty liver disease. Hepatology. 2012;55(1):77-85.

6. Zhou F, Yu T, Du R, Fan G, Liu Y, Liu Z, Xiang J, Wang Y, Song B, Gu X, Guan L, Wei Y, Li H, Wu X, Xu J, Tu S, Zhang Y, Chen H, Cao B. Clinical course and risk factors for mortality of adult inpatients with COVID-19 in Wuhan, China: a retrospective cohort study. Lancet. 2020;395(10229):1054-62.

7. Chen G, Wu D, Guo W, Cao Y, Huang D, Wang H, Wang T, Zhang X, Chen H, Yu H, Zhang X, Zhang M, Wu S, Song J, Chen T, Han M, Li S, Luo X, Zhao J, Ning Q. Clinical and immunologic features in severe and moderate Coronavirus Disease 2019. J Clin Invest 2020: [Epub ahead of print].

8. National Health Commission of the People's Republic of China. Diagnosis and treatment of novel coronavirus pneumonia (the seventh edition).

http://www.nhc.gov.cn/yzygj/s7653p/202003/46c9294a7dfe4cef80dc7f5912eb1989/files/ce3e6945832a438eaae415350a8ce964.pdf. March 42020.

9. Tan L, Wang Q, Zhang D, Ding J, Huang Q, Tang YQ, Wang Q, Miao H. Lymphopenia predicts disease severity of COVID-19: a descriptive and predictive study. Signal Transduct Target Ther. 2020;5:33.

10. Liu Y, Du X, Chen J, Jin Y, Peng L, Wang HHX, Luo M, Chen L, Zhao Y. Neutrophil-to-lymphocyte ratio as an independent risk factor for mortality in hospitalized patients with COVID-19. J Infect 2020: [Epub ahead of print].

11. Luo X, Zhou W, Yan X, Guo T, Wang B, Xia H, Ye L, Xiong J, Jiang Z, Liu Y, Zhang B, Yang W. Prognostic value of C-reactive protein in patients with COVID-19. medRxiv preprint 2020.

12. Jordan RE, Adab P, Cheng KK. Covid-19: risk factors for severe disease and death. BMJ. 2020;368:m1198.

13. Huang C, Wang Y, Li X, Ren L, Zhao J, Hu Y, Zhang L, Fan G, Xu J, Gu X, Cheng Z, Yu T, Xia J, Wei Y, Wu W, Xie X, Yin W, Li H, Liu M, Xiao Y, Gao H, Guo L, Xie J, Wang G, Jiang R, Gao Z, Jin Q, Wang J, Cao B. Clinical features of patients infected with 2019 novel coronavirus in Wuhan, China. Lancet. 2020;395(10223):497-506.

14. Du RH, Liang LR, Yang CQ, Wang W, Cao TZ, Li M, Guo GY, Du J, Zheng CL, Zhu Q, Hu M, Li XY, Peng P, Shi HZ. Predictors of Mortality for Patients with COVID-19 Pneumonia Caused by SARS-CoV-2: A Prospective Cohort Study. Eur Respir J 2020: [Epub ahead of print].

15. Chen X, Hu W, Ling J, Mo P, Zhang Y, Jiang Q, Ma Z, Cao Q, Deng L, Song S, Zheng R, Gao S, Ke H, Gui X, Lundkvist Ak, Li J, Lindahl J, Xiong Y. Hypertension and Diabetes Delay the Viral Clearance in COVID-19 Patients. medRxiv preprint 2020.

16. Koperdanova M, Cullis JO. Interpreting raised serum ferritin levels. BMJ. 2015;351:h3692.

17. Trombini P, Piperno A. Ferritin, metabolic syndrome and NAFLD: elective attractions and dangerous liaisons. Journal of hepatology 2007: 46(4): 549-552.

18. Bozzini C, Girelli D, Olivieri O, Martinelli N, Bassi A, De Matteis G, Tenuti I, Lotto V, Friso S, Pizzolo F, Corrocher R. Prevalence of body iron excess in the metabolic syndrome. Diabetes Care. 2005;28(8):2061-3.

19. Zhang C, Shi L, Wang FS. Liver injury in COVID-19: management and challenges. Lancet Gastroenterol Hepatol 2020: 5(5): 428-430.

\section{Tables}

Table 1. Demographic and characteristics of COVID-19 patients ${ }^{\text {a }}$ 


\begin{tabular}{|c|c|c|c|c|}
\hline & Total & Normal ferritin group $\square \leq 200 \mathrm{ng} / \mathrm{mL} \square$ & Elevation ferritin group $\square>200 \mathrm{ng} / \mathrm{mL} \square$ & p-value \\
\hline & $\mathrm{n}=79$ & $\mathrm{n}=35$ & $\mathrm{n}=44$ & \\
\hline Severe patient & $23(29.1 \%)$ & $1(2.9 \%)$ & $22(50.0 \%)$ & $<0.001$ \\
\hline \multicolumn{5}{|l|}{ Baseline } \\
\hline Age (years) & $44.1 \pm 14.4$ & $36.8 \pm 12.6$ & $49.9 \pm 13.1$ & $<0.001$ \\
\hline Sex (male) & $36(45.6 \%)$ & $6(17.1 \%)$ & $30(68.2 \%)$ & $<0.001$ \\
\hline Days from illness onset to admission, day & $8.5(5.0-15.0)$ & $10.0(5.0-15.0)$ & $7.0(5.5-14.5)$ & 0.324 \\
\hline SBP (mmHg) & $126.5 \pm 16.0$ & $122.5 \pm 15.9$ & $130.5 \pm 15.3$ & 0.048 \\
\hline DBP (mmHg) & $79.2 \pm 10.4$ & $77.6 \pm 9.4$ & $80.7 \pm 11.2$ & 0.244 \\
\hline Respire (breaths per min) & $20.1 \pm 1.0$ & $20.1 \pm 1.1$ & $20.1 \pm 0.9$ & 0.894 \\
\hline Pulse (beats per min) & $84.7 \pm 10.7$ & $84.0 \pm 9.8$ & $85.4 \pm 11.7$ & 0.602 \\
\hline Smoking (yes) & $3(3.8 \%)$ & $3(8.6 \%)$ & $0(0.0 \%)$ & 0.048 \\
\hline Disease severity status & & & & $<0.001$ \\
\hline Severe & $23(29.1 \%)$ & $1(2.9 \%)$ & $22(50.0 \%)$ & \\
\hline Non- severe & $56(70.9 \%)$ & $34(97.1 \%)$ & $22(50.0 \%)$ & \\
\hline \multicolumn{5}{|l|}{ Comorbidities } \\
\hline Diabetes & $9(11.4 \%)$ & $5(14.3 \%)$ & $4(9.1 \%)$ & 0.470 \\
\hline Hypertension & $14(17.7 \%)$ & $7(20.0 \%)$ & $7(15.9 \%)$ & 0.636 \\
\hline Others & $27(34.2 \%)$ & $9(25.7 \%)$ & $18(40.9 \%)$ & 0.157 \\
\hline \multicolumn{5}{|l|}{ Symptoms } \\
\hline Fever & $59(74.7 \%)$ & $23(65.7 \%)$ & $36(81.8 \%)$ & 0.102 \\
\hline Cough & $39(49.4 \%)$ & $17(48.6 \%)$ & $22(50.0 \%)$ & 0.900 \\
\hline Fatigue & $20(25.3 \%)$ & $8(22.9 \%)$ & $12(27.3 \%)$ & 0.654 \\
\hline Sore muscle & $8(10.1 \%)$ & $6(17.1 \%)$ & $2(4.5 \%)$ & 0.065 \\
\hline Diarrhea & $2(2.5 \%)$ & $0(0.0 \%)$ & $2(4.5 \%)$ & 0.201 \\
\hline Chest distress & $11(13.9 \%)$ & $6(17.1 \%)$ & $5(11.4 \%)$ & 0.461 \\
\hline Expectoration & $6(7.6 \%)$ & $4(11.4 \%)$ & $2(4.5 \%)$ & 0.251 \\
\hline Sore throat & $5(6.3 \%)$ & $3(8.6 \%)$ & $2(4.5 \%)$ & 0.465 \\
\hline Headache & $4(5.1 \%)$ & $2(5.7 \%)$ & $2(4.5 \%)$ & 0.814 \\
\hline Anhelation & $15(19.0 \%)$ & $5(14.3 \%)$ & $10(22.7 \%)$ & 0.342 \\
\hline \multicolumn{5}{|l|}{ Drug Treatment } \\
\hline Antivirus & $76(96.2 \%)$ & $33(94.3 \%)$ & $43(97.7 \%)$ & 0.427 \\
\hline Antibacteria & $67(84.8 \%)$ & $28(80.0 \%)$ & $39(88.6 \%)$ & 0.288 \\
\hline Expectorant & $30(38.0 \%)$ & $11(31.4 \%)$ & $19(43.2 \%)$ & 0.285 \\
\hline Hormone & $13(16.5 \%)$ & $3(8.6 \%)$ & $10(22.7 \%)$ & 0.092 \\
\hline Hepatinica & $18(22.8 \%)$ & $6(17.2 \%)$ & $12(27.3 \%)$ & 0.286 \\
\hline Chinese medicine & $16(20.3 \%)$ & $5(14.3 \%)$ & $11(25.0 \%)$ & 0.239 \\
\hline Immune Globulin & $19(24.1 \%)$ & $8(22.9 \%)$ & $11(25.0 \%)$ & 0.825 \\
\hline Outcome & & & & 0.011 \\
\hline Discharged & $69(87.3 \%)$ & $35(100.0 \%)$ & $34(77.3 \%)$ & \\
\hline Death & $2(2.5 \%)$ & $0(0.00 \%)$ & $2(4.5 \%)$ & \\
\hline Transfer to other hospital & $8(10.1 \%)$ & $0(0.00 \%)$ & $8(18.2 \%)$ & \\
\hline
\end{tabular}

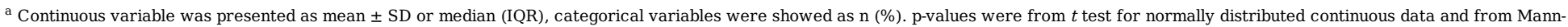
Whitney U test for abnormally distributed continuous data. p-values were from $\chi$ ¿test for categorical data.

Figures 


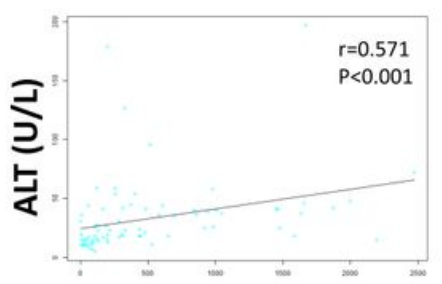

Ferritin $(\mathrm{ng} / \mathrm{mL}$ )

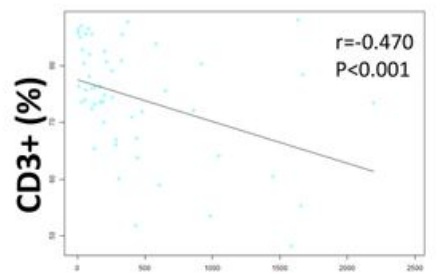

Ferritin $(\mathrm{ng} / \mathrm{mL})$

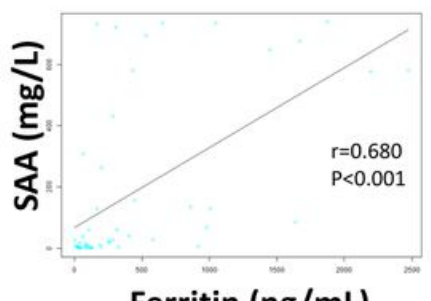

Ferritin $(\mathrm{ng} / \mathrm{mL}$ )

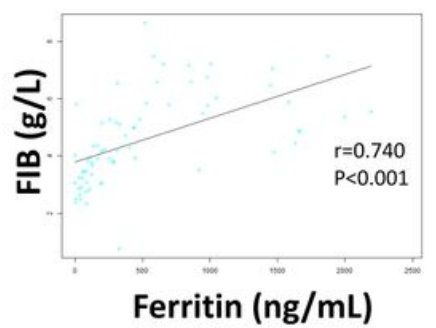

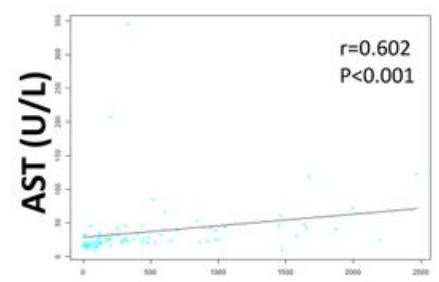

Ferritin $(\mathrm{ng} / \mathrm{mL})$
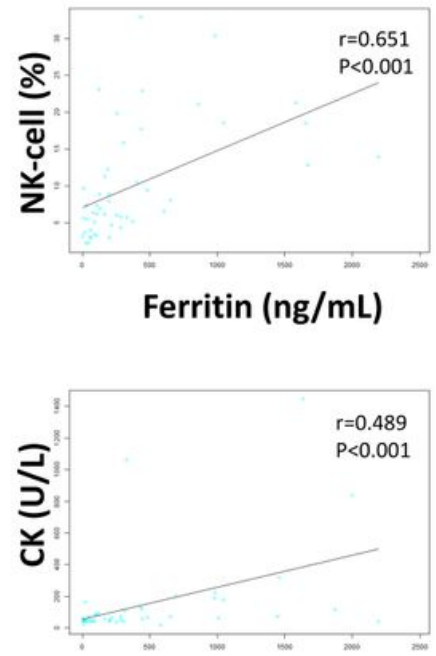

Ferritin $(\mathrm{ng} / \mathrm{mL})$

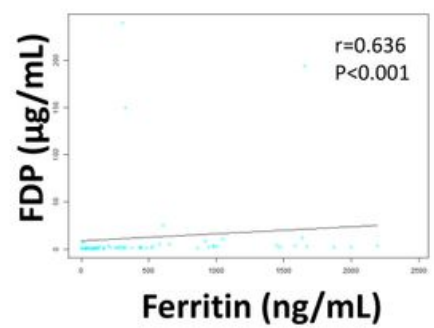

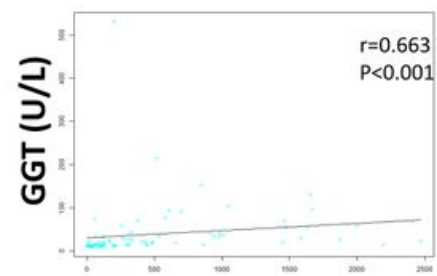

Ferritin $(\mathrm{ng} / \mathrm{mL})$
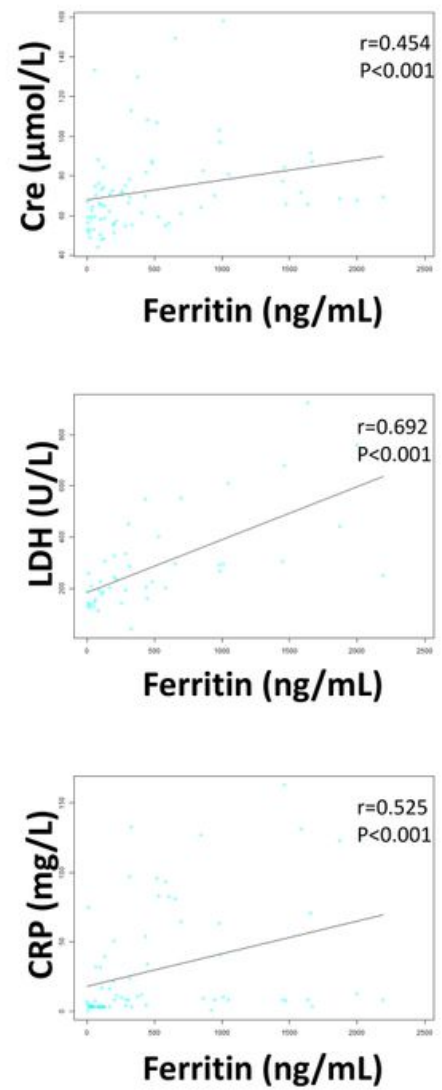

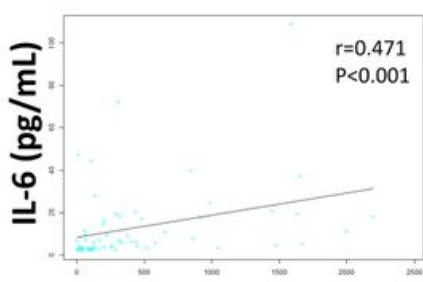

Ferritin $(\mathrm{ng} / \mathrm{mL})$

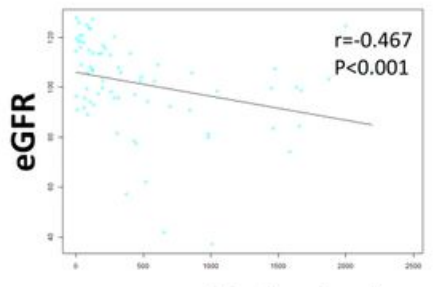

Ferritin $(\mathrm{ng} / \mathrm{mL})$

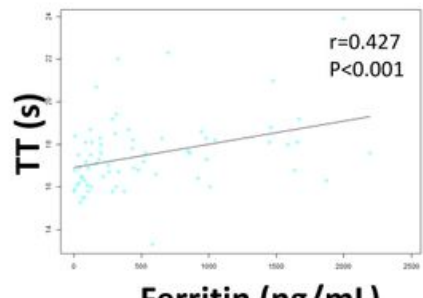

Ferritin $(\mathrm{ng} / \mathrm{mL})$

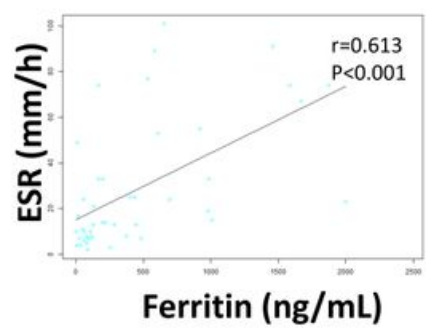

Figure 1

Correlation between ferritin and other serum biomarkers. 

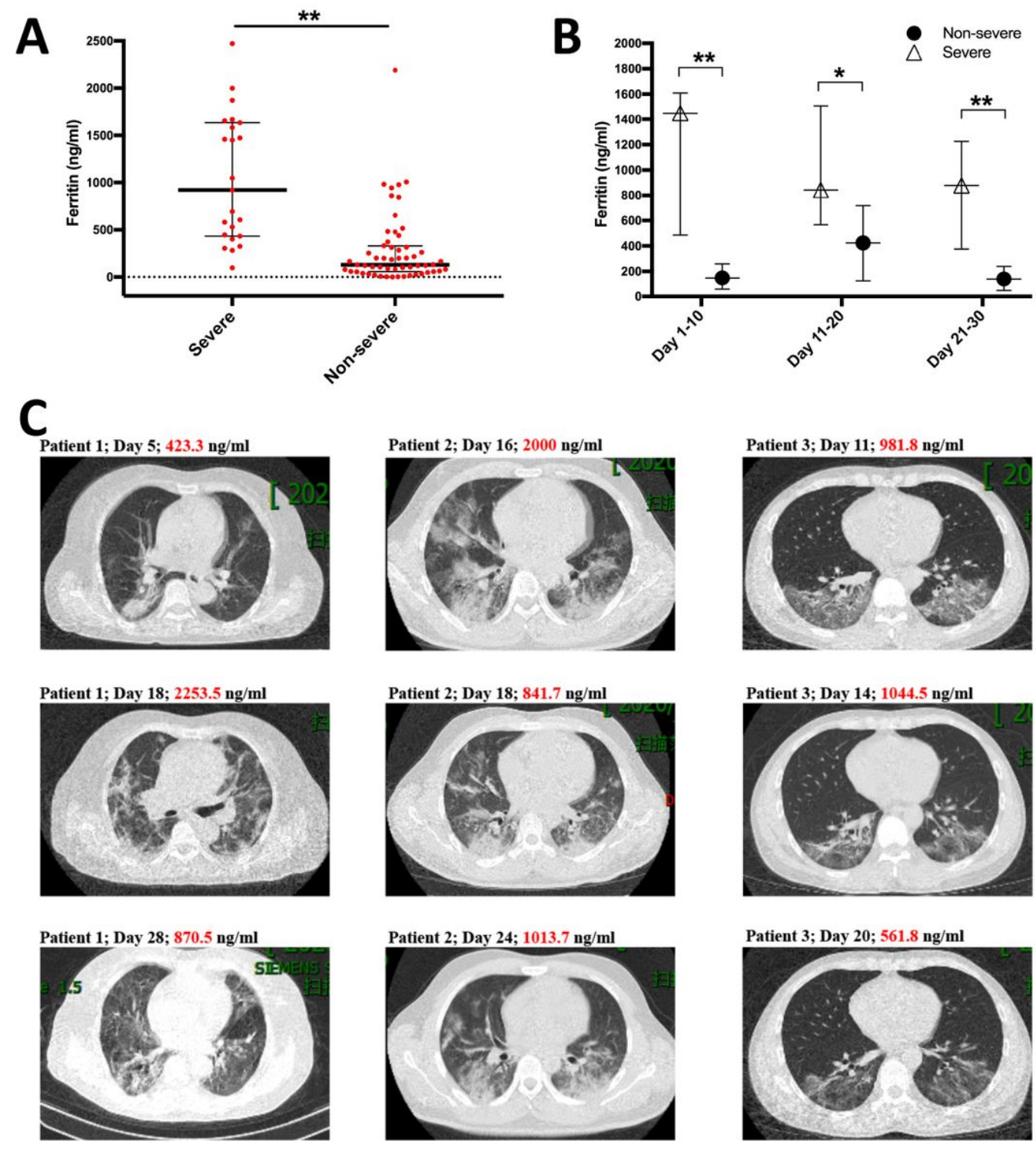

Figure 2

(A) The levels of ferritin in severe and non-severe patients on admission; (B) The monitor of ferritin calculated from the onset of symptom; (C) CT manifestation of severe (patient 1\&2) and non-severe (patient 3) cases (the corresponding ferritin levels and time points are displayed on the top of each image). The data are shown as median (IQR); ** $p<0.001,{ }^{*} p<0.01$. 


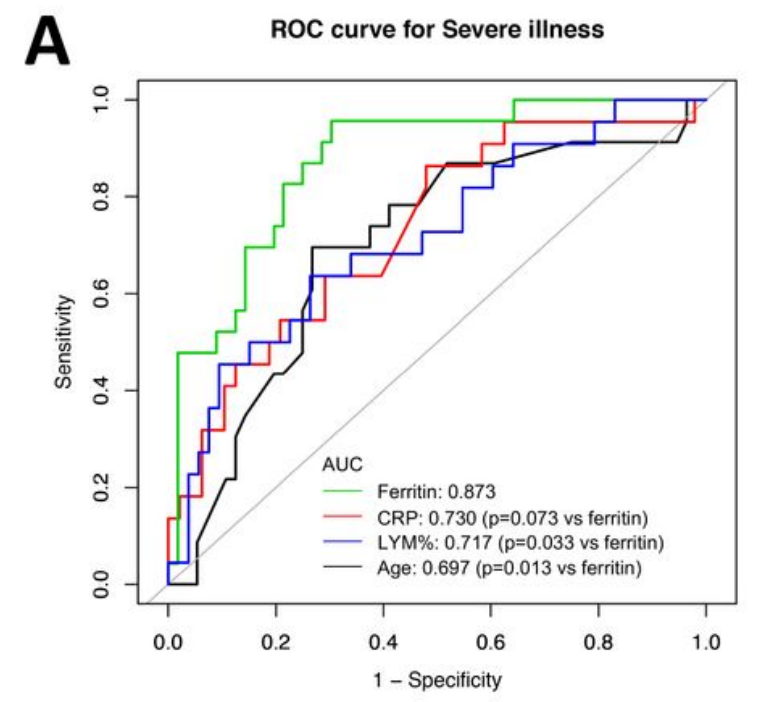

C Ferritin per $100 \mathrm{ng} / \mathrm{ml}$ increase

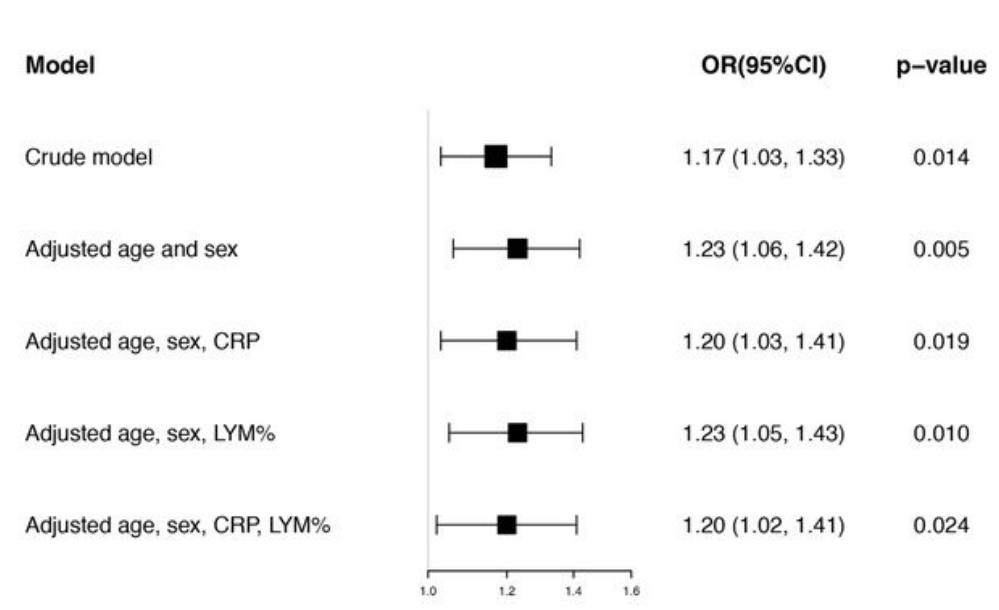

E

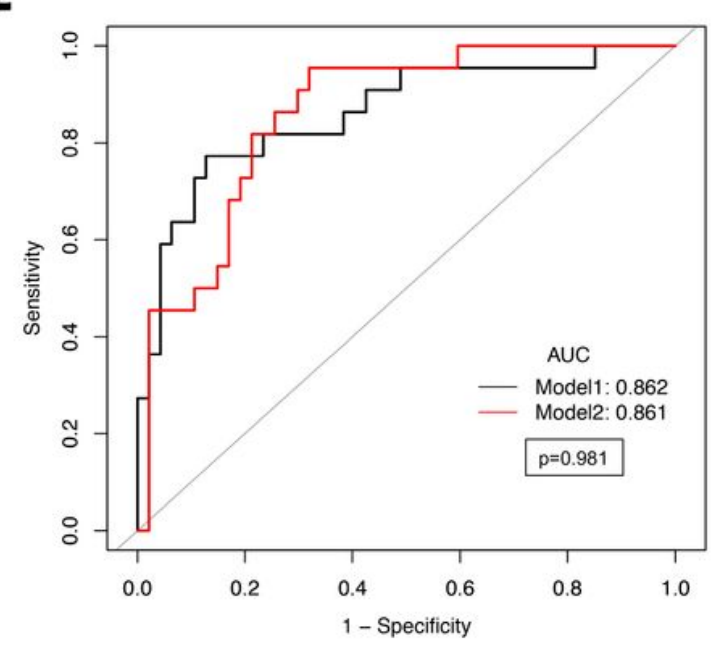

\section{D}

Ferritin $>=200$ VS $<200$ ng/ml

Model

Crude model

Adjusted age and sex

Adjusted age, sex, CRP

Adjusted age, sex, LYM\%

Adjusted age, sex, CRP, LYM\%
OR( $(95 \% \mathrm{Cl})$

p-value

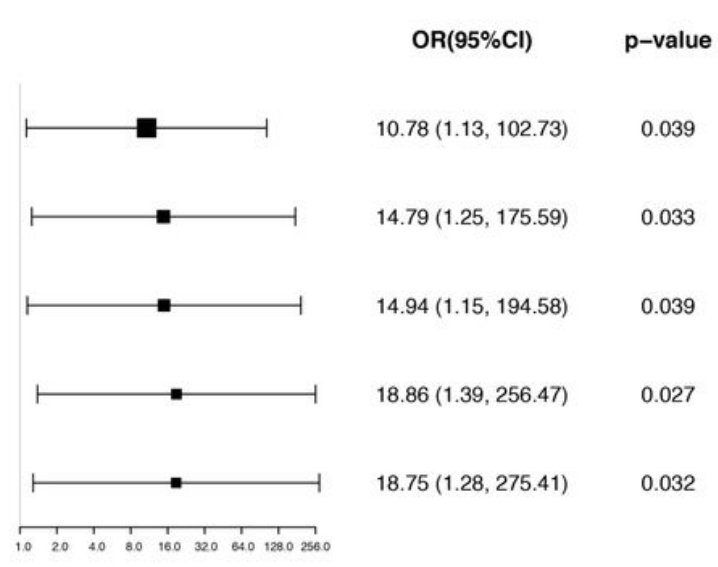

Figure 3

(A) ROC curves of potential risk factors ( $n=79$ for age and ferritin, $n=75$ for LYM\%, $n=70$ for CRP); (B) The comparison of ROC curves between ferritin alone and multivariable model ( $n=69$, Model 1: ferritin independent factor, Model 2: multiple factors integrating gender, age, ferritin, LYM\% and CRP); (C) Forest plots of logistic regression analysis between serum ferritin (assessed as a continuous variable, per $100 \mathrm{ng} / \mathrm{mL}$ ) and the risk of severity illness; (D) Forest plots of logistic regression analysis between serum ferritin (assessed as a categorical variable, $\leq 200 \mathrm{ng} / \mathrm{mL}$ and $>200 \mathrm{ng} / \mathrm{mL}$ ) and the risk of severity illness. 
Points

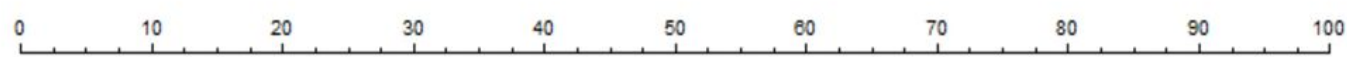

Age $\quad \begin{aligned} \text { nाmmmm } \\ 20 \quad 65\end{aligned}$

Sex

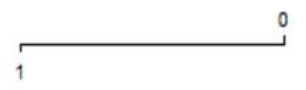

CRP

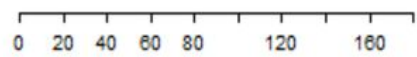

LYM\%

\begin{tabular}{lllllllllllll}
\hline 55 & 50 & 45 & 40 & 35 & 30 & 25 & 20 & 15 & 10 & 5 & 0
\end{tabular}

Ferritin

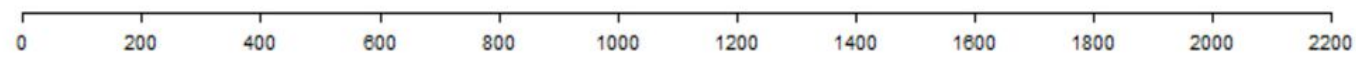

Total points

(20

20

60

80

100

120

140

180

Linear predictor

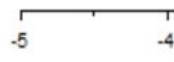

$+3$

$-2$

Severe illness

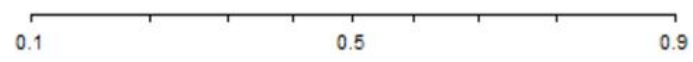

Figure 4

Nomogram incorporating risk factors.

A
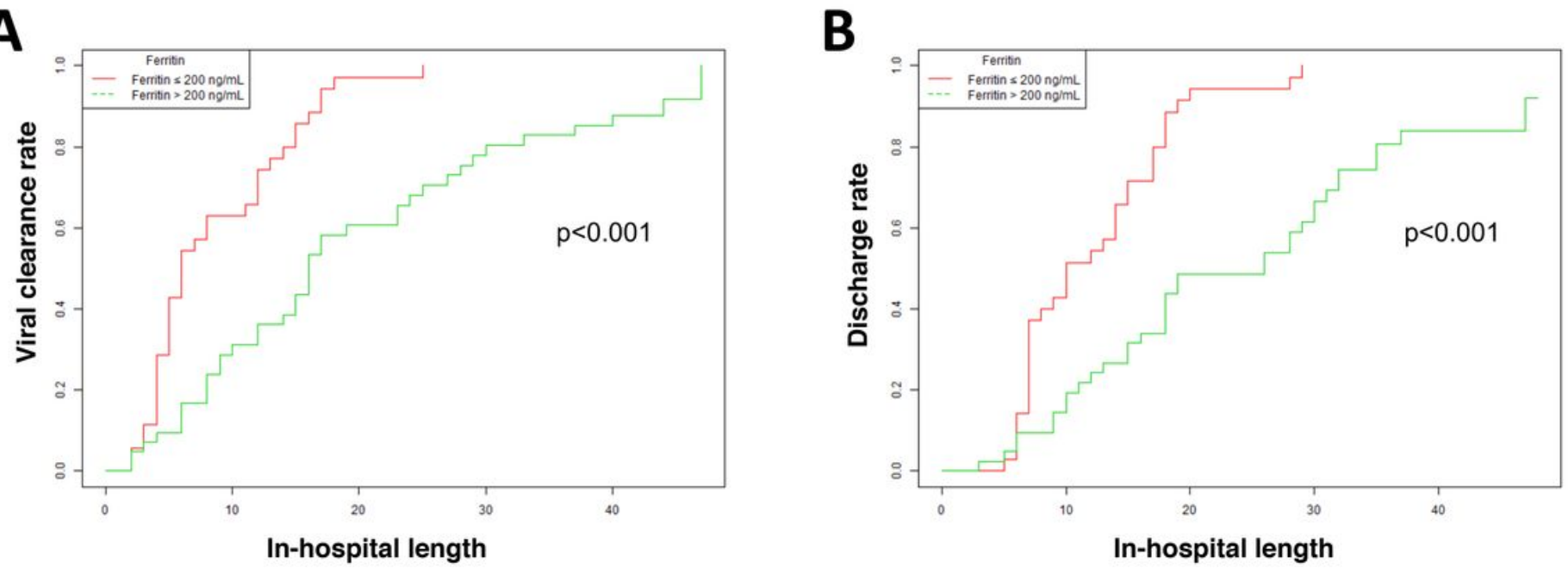

Figure 5

Page $11 / 12$ 
Kaplan Meier estimates of viral clearance rate $(A)$ and inpatient discharge rate $(B)$ over time stratified by serum ferritin levels. (77 patients were included except 2 deceased ones)

\section{Supplementary Files}

This is a list of supplementary files associated with this preprint. Click to download.

- SupplementalTable.docx 